\title{
ANÁLISE DAS POSSIBILIDADES DE TRANSFORMAÇÃO DE REFERENCIAL E ATUALIZAÇÃO DE COORDENADAS ESTIMADAS PELO PPP
}

\author{
Analysis of possibilities of referential transformation and update of estimated \\ coordinates using PPP
}

Marcony de Paulo Ramos ${ }^{1}$

William Rodrigo Dal Poz ${ }^{1,2}$

Alessandro Salles Carvalho ${ }^{1,3}$

1 Universidade Federal de Viçosa - UFV. Programa de Pós-Graduação em Engenharia Civil.
2 Universidade Federal de Viçosa - UFV. Departamento de Engenharia Civil. Setor de Engenharia de
Agrimensura e Cartografia.
${ }^{3}$ Universidade Federal de Juiz de Fora - UFJF.Juiz de Fora - Minas Gerais - Brasil.
Email:marcony.ramos@ufv.br; william.dalpoz@ufv.br; alessandro.carvalho@ufjf.edu.br

\section{Resumo:}

O sistema de referência oficial adotado no Brasil é o SIRGAS2000 (densificação do ITRF2000) e foi realizado na época 2000,4. Desde a semana GPS 1709 (07/10/2012), o referencial adotado pelo IGS (International GNSS Service) para as efemérides precisas é o IGb08 (ITRF2008). Desse modo, os serviços que realizam o Posicionamento por Ponto Preciso (PPP) e empregam essas efemérides no processamento fornecem as coordenadas das estações nesse referencial e na época de coleta dos dados. Portanto, torna-se necessário realizar a transformação das coordenadas do IGb08 (ITRF2008, época de coleta dos dados) para o SIRGAS2000 (ITRF2000, época 2000,4). Para realizar essa tarefa existem, pelo menos, 45 possibilidades, pois há pelo menos 3 conjuntos de parâmetros de transformação que podem ser usados em conjunto com pelo menos 15 modelos de velocidade. O objetivo deste trabalho é avaliar essas possibilidades no IBGE-PPP. Foram processados dados de 86 estações da RBMC (Rede Brasileira de Monitoramento Contínuo dos Sistemas GNSS) coletados no dia (15/07/2013). Os resultados mostram que a melhor possibilidade de transformação de referencial e atualização de coordenadas, na média, é aquela que utiliza os parâmetros de transformação disponibilizados por Petit e Luzum (2010), juntamente com o modelo de velocidade MORVEL2010.

Palavras-chave: IBGE-PPP, campo de velocidade, GNSS, parâmetros de transformação.

\section{Abstract:}

The official reference system adopted in Brazil is SIRGAS2000 (ITRF2000 densification) and was materialized at the epoch 2000,4. Since the GPS week 1709 (07/10/2012), the IGS (International GNSS Service) reference frame, including IGS ephemerides, is the IGb08 (ITRF2008). Thus, the online PPP services provide the coordinates of the GNSS stations in IGb08, at the epoch of GNSS data collection. Therefore, it becomes necessary to perform the transformation of IGb08 reference frame (ITRF2008, epoch of GNSS data collection) for 
SIRGAS2000 (ITRF2000, epoch 2000.4). For this purpose, there are at least 45 possibilities, because there are at least 3 sets of transformation parameters that can be combined with at least 15 velocity models. The objective of this paper is to evaluate these possibilities in the IBGE-PPP. To accomplish this task, were processed GNSS data of 86 stations of Permanent GNSS Network of Brazil $(15 / 07 / 2013)$. The results show that the best possibility of transformation between reference frames and coordinate update, on average, is one that uses the transformation parameters supplied by Luzum and Petit (2010), combined with the velocity field model MORVEL2010.

Keywords: IBGE-PPP, velocity field, GNSS, transformation parameters.

\section{Introdução}

O processamento dos dados GNSS (Global Navigation Satellite System) com base no método PPP (Posicionamento por Ponto Preciso) é realizado, de modo geral, utilizando serviços gratuitos disponíveis na Web. As efemérides precisas dos satélites dos sistemas GPS (Global Positioning System) e GLONASS (Global'naya Navigatsionnaya Sputnikkovaya Sistema) empregadas nesses serviços são fornecidas pelo IGS (International GNSS Service) e pelo CDDIS (Crustal Dynamics Data Information System) e estão referenciadas ao sistema IGb08 desde a semana GPS 1709 (07/10/2012). Salienta-se que este sistema tem como base o ITRF2008 (International Terrestrial Reference Frame 2008). O IGb08 foi obtido somente com o emprego de observações GNSS, enquanto no ITRF2008 também foram utilizadas as técnicas de posicionamento VLBI (Very Long Baseline Interferometry), SLR (Satellite Laser Ranging) e DORIS (Doppler Orbitography and Radiopositioning Integrated on Satellite). Contudo, ressalta-se que o ITRF2008 e o IGb08 são alinhados, pois compartilham a mesma origem, escala e orientação (EPNCB, 2014).

No Brasil, o sistema geodésico oficial é o SIRGAS2000 e as coordenadas dos satélites GNSS estão, atualmente, referenciadas ao IGb08, então as coordenadas estimadas no serviço de PPP online estarão nesse referencial, na época de coleta dos dados. Devido a diferença de referencial e época das coordenadas das estações fornecidas pelos serviços PPP e o adotado no Brasil para posicionamentos geodésicos, torna-se necessário o emprego de parâmetros da transformação de Helmert e um modelo de velocidade para compatibilização de referencial e época. Ressalta-se que o sistema de referência oficial do Brasil, ou seja, o SIRGAS2000, é uma densificação regional do ITRF2000, na época 2000,4 (SIRGAS, 2015).

Atualmente existem vários serviços de posicionamento online, tais como: CSRS-PPP, disponibilizado pelo NRCan (Natural Resources Canada); IBGE-PPP (Instituto Brasileiro de Geografia e Estatística - PPP) que faz o uso do aplicativo de processamento CSRS_PPP; GAPS (GPS Analysis and Positioning Software), da UNB (University of New Brunswick); APPS (Automatic Precise Positioning Service) do JPL (Jet Propulsion Laboratory); magicGNSS da GMV Aerospace and Defense; AUSPOS (Online GPS Processing) fornecido pela GA (Geoscience Australia); SCOUT (Scripps Coordinate Update Tool) desenvolvido pela SOPAC (Scripps Orbit and Permanent Array Center); e OPUS (Online Positioning User Service) desenvolvido pelo NGS (United States' National Geodetic Survey).

O IBGE-PPP foi adaptado para uso no Brasil, possibilitando os usuários de GNSS obterem coordenadas precisas e referenciadas ao SIRGAS2000 (Sistema de Referência Geocêntrico para as Américas 2000) e ao IGb08, tanto na época do levantamento, quanto na data da realização SIRGAS2000, época 2000,4. 
Na realização da mudança de referencial das coordenadas das estações, o IBGE-PPP emprega sete parâmetros da transformação de Helmert (três translações, três rotações e um fator escala) que são disponibilizados no arquivo *.SUM, presente nos resultados do processamento. A atualização das coordenadas no IBGE-PPP é realizada com base nas velocidades obtidas com o uso do modelo VEMOS2009 (VEelocity MOdel for Sirgas). Detalhes sobre este procedimento, realizado pelo IBGE, no processo de mudança de referencial e atualização de coordenadas podem ser encontrados em Carvalho et al. (2015).

Além dos parâmetros de transformação disponibilizados no relatório do IBGE-PPP para mudança de referencial das coordenadas do IGb08 (ITRF2008) para o SIRGAS2000 há parâmetros estimados por Petit e Luzum (2010) e por Altamimi et al.(2007). Nos parâmetros estimados por Petit e Luzum (2010), a mudança de referencial é realizada de forma direta do ITRF2008 para o ITRF2000; nos fornecidos por Altamimi et al.(2007) é executada com um transformação intermediária, ou seja, do ITRF2008 para ITRF2005 e deste para o ITRF2000.

Há também vários outros modelos de velocidade disponíveis, tais como APKIM 2000, APKIM 2005 DGFI, APKIM2005 IGN, CGPS 2004, GEODVEL 2010, GSRM_V1, HS2-NUVEL 1A, HS3-NUVEL 1A, ITRF2000 ASB, ITRF2000 DA, MORVEL 2010, NUVEL 1, NUVEL 1A e REVEL 2000 (14 no total); são pelo menos 15 modelos, incluindo o VEMOS2009. Alguns desses modelos, são originados de observações de geodésia espacial, outros, gerados a partir de dados geológicos e geofísicos. Informações sobre estes modelos podem ser encontradas em UNAVCO (2014).

Diante do exposto, verifica-se a existência de diferentes possibilidades de transformação de referencial e atualização de coordenadas e a necessidade de compatibilização de referencial e época. Tem-se pelo menos 45 possibilidades, pois há 3 conjuntos de parâmetros de transformação (considerando também os parâmetros do IBGE) que podem ser usados em conjunto com 15 modelos de velocidade, totalizando as 45 possibilidades. Portanto, este trabalho tem por objetivo avaliar a qualidade do posicionamento planimétrico com base nessas possibilidades de transformação de referencial e atualização das coordenadas obtidas com uso do IBGE-PPP, de modo que seja possível eleger a melhor combinação de parâmetros de transformação e modelo de velocidades para o Brasil. Salienta-se que as metodologias utilizadas neste trabalho podem ser aplicadas em qualquer outro serviço de posicionamento online.

\section{Parâmetros de Transformação}

Como as coordenadas dos satélites GNSS estão, atualmente, referenciadas ao IGb08 (efemérides precisas), então as coordenadas estimadas no serviço de PPP online estarão nesse referencial, na época de coleta dos dados. Contudo, no Brasil, desde 25/02/2015, o SIRGAS2000 passou a ser oficialmente o único sistema geodésico de referência no Brasil (IBGE, 2015b). Desta forma, as coordenadas estimadas em IGb08, época de coleta dos dados, devem ser transformadas e atualizadas para o SIRGAS2000, época 2000,4. Como já descrito, isto pode ser realizado a partir de pelo menos 45 possibilidades, em combinação com os parâmetros de transformação (3 parâmetros) e modelos de velocidade mencionados (15 modelos).

Para transformação de referencial tem-se os parâmetros utilizados pelo IBGE, aplicados no IBGE-PPP, e mais dois conjuntos de parâmetros disponibilizados por Petit e Luzum (2010) e Altamimi et al. (2007). Ao aplicar estes dois últimos conjuntos de parâmetros de transformação 
no IBGE-PPP, são consideradas as compatibilidades entre o IGb08 e o ITRF2008 e entre o ITRF2000 e o SIRGAS2000.

Os parâmetros de transformação consistem de três translações, três rotações, um fator de escala e suas respectivas variações temporais (rates). As translações são fornecidas em milímetros, o fator de escala (s) em partes por bilhão e as rotações em milissegundos de arco. As variações temporais das translações são dados em milímetros por ano, do fator de escala em partes por bilhão por ano e das rotações em miliarcossegundo por ano. A Tabela 1 apresenta os parâmetros de transformação do ITRF2008 para os ITRF2005 e ITRF2000; e do ITRF2005 para o ITRF2000.

Tabela 1: Parâmetros de transformação de referencial disponibilizados pelo ITRF.

\begin{tabular}{|c|c|c|c|c|c|c|c|}
\hline Parâmetros $\Rightarrow$ & $\underset{\mathrm{mm}}{\mathrm{T}_{\mathbf{x}}}$ & $\underset{\mathbf{m m}}{\mathbf{T}_{\mathrm{y}}}$ & $\begin{array}{c}\mathbf{T}_{2} \\
\mathbf{m m}\end{array}$ & $\underset{\mathrm{ppb}}{\mathrm{S}}$ & $\varepsilon_{\mathrm{v}}$ & $\begin{array}{c}\varepsilon_{y} \\
\text { mas }\end{array}$ & $\begin{array}{c}\varepsilon_{\mathrm{x}} \\
\text { mas }\end{array}$ \\
\hline Variação $\Rightarrow$ & $\underset{\mathrm{mm} / \mathrm{a}}{\mathrm{T}_{\mathrm{x}}}$ & $\underset{\mathrm{mm} / \mathbf{a}}{\mathrm{T}_{\mathrm{y}}}$ & $\begin{array}{c}\mathrm{T}_{2} \\
\mathrm{~mm} / \mathbf{a}\end{array}$ & $\underset{\mathrm{ppb} / \mathrm{a}}{\mathrm{S}}$ & $\varepsilon_{\mathrm{mas} / \mathrm{a}}^{\varepsilon_{\mathrm{v}}}$ & $\begin{array}{c}\varepsilon_{y} \\
\mathrm{mas} / \mathrm{a}\end{array}$ & $\begin{array}{c}\varepsilon_{\mathrm{z}} \\
\mathrm{m} \text { as/a }\end{array}$ \\
\hline $\begin{array}{l}\text { ITRF } 2005 \Rightarrow \text { ITRF } 2000 \\
\text { (época 2000,0) }\end{array}$ & $\begin{array}{r}0,1 \\
-0,2\end{array}$ & $\begin{array}{r}-0,8 \\
0,1\end{array}$ & $\begin{array}{l}-5,8 \\
-1,80\end{array}$ & $\begin{array}{l}0,40 \\
0,08\end{array}$ & $\begin{array}{l}0,00 \\
0,00\end{array}$ & $\begin{array}{l}0,00 \\
0,00\end{array}$ & $\begin{array}{l}0,00 \\
0,00\end{array}$ \\
\hline $\begin{array}{l}\text { ITRF2008 } \Rightarrow \text { ITRF2005 } \\
\quad \text { (época 2005,0) }\end{array}$ & $\begin{array}{r}-0,5 \\
0,3\end{array}$ & $\begin{array}{r}-0,9 \\
0,0\end{array}$ & $\begin{array}{l}-4,7 \\
-0,0\end{array}$ & $\begin{array}{l}0,94 \\
0,00\end{array}$ & $\begin{array}{l}0,00 \\
0,00\end{array}$ & $\begin{array}{l}0,00 \\
0,00\end{array}$ & $\begin{array}{l}0,00 \\
0,00\end{array}$ \\
\hline $\begin{array}{l}\text { ITRF2008 } \Rightarrow \text { ITRF } 2000 \\
\text { (época } 2000,0)\end{array}$ & $\begin{array}{r}-1,9 \\
0,1 \\
\end{array}$ & $\begin{array}{r}-1,7 \\
0,1 \\
\end{array}$ & $\begin{array}{l}-10,5 \\
-1,8\end{array}$ & $\begin{array}{l}1,34 \\
0,08\end{array}$ & $\begin{array}{l}0,00 \\
0,00\end{array}$ & $\begin{array}{l}0,00 \\
0,00\end{array}$ & $\begin{array}{l}0,00 \\
0,00\end{array}$ \\
\hline
\end{tabular}

Fonte: Petit e Luzum.(2010); Altamimi. et al.(2007).

Para realizar a mudança de referencial IGb08(ITRF2008) para o sistema SIRGAS2000 (ITRF2000), o IBGE disponibiliza os parâmetros de transformação de referencial. O relacionamento entre essas realizações também se dá através de uma transformação de 7 parâmetros (três translações, três rotações e escala) que foram estimados através de coordenadas de estações GNSS permanentes localizadas no continente Sul-Americano e presentes nas realizações ITRF2008 e SIRGAS2000 (IBGE, 2013). A Tabela 2 contém os parâmetros de transformação que relacionam o IGb08(ITRF2008) e o SIRGAS2000 (IBGE, 2013) e que são utilizados pelo IBGE.

Tabela 2: Parâmetros de transformação do IGb08 (ITRF2008) para o ITRF2000(SIRGAS2000).

\begin{tabular}{|c|c|c|c|c|c|c|c|}
\hline Parâmetros $\Rightarrow$ & $\begin{array}{c}\mathrm{T}_{\mathrm{x}} \\
\mathrm{mm}\end{array}$ & $\begin{array}{c}\mathrm{T}_{\mathrm{y}} \\
\mathrm{mm}\end{array}$ & $\begin{array}{c}\mathbf{T}_{\mathbf{z}} \\
\mathrm{mm}\end{array}$ & $\begin{array}{c}\mathrm{S} \\
\mathrm{ppb}\end{array}$ & $\begin{array}{c}\boldsymbol{\varepsilon}_{\mathbf{Y}} \\
\text { mas }\end{array}$ & $\begin{array}{c}\varepsilon_{\mathrm{y}} \\
\text { mas }\end{array}$ & $\begin{array}{c}E_{2} \\
\text { mas }\end{array}$ \\
\hline Variação $\Rightarrow$ & $\begin{array}{c}T_{x} \\
\mathrm{~m} / \mathbf{a}\end{array}$ & $\begin{array}{c}\mathbf{T}_{y} \\
\mathrm{~m} / \mathbf{a}\end{array}$ & $\begin{array}{c}\mathrm{T}_{\mathrm{z}} \\
\mathrm{m} / \mathbf{a}\end{array}$ & $\begin{array}{c}\mathrm{S} \\
\mathrm{ppb} / \mathrm{a}\end{array}$ & $\begin{array}{c}\varepsilon_{\mathrm{v}} \\
\mathrm{mas} / \mathrm{a}\end{array}$ & $\begin{array}{c}\boldsymbol{\varepsilon}_{\mathrm{y}} \\
\mathrm{mas} / \mathbf{a}\end{array}$ & $\begin{array}{c}\varepsilon_{v_{7}} \\
\text { mas/a }\end{array}$ \\
\hline IGb08 $\Rightarrow$ SIRGAS 2000 & 2 & 4,1 & 3,9 & $-1,00$ & 0,17 & $-0,03$ & 0,070 \\
\hline & 0,000 & 0,000 & 0,000 & 0,00 & 0,00 & 0,00 & 0,000 \\
\hline
\end{tabular}

Fonte: IBGE (2013).

Nota-se na Tabela 2 que os valores dos parâmetros adotados pelo IBGE-PPP são invariantes no tempo, pois as variações temporais são nulas; ao contrário do que ocorrem com os parâmetros disponibilizados em Petit e Luzum (2010) e Altamimi et al. (2007). 


\section{Transformação de Referencial e Atualização de Coordenadas}

O processo de mudança de referencial e atualização das coordenadas pode envolver três épocas: época de origem $\left(\mathrm{t}_{0}\right)$, época destino ou de interesse $(\mathrm{t})$ e época dos parâmetros de transformação $\left(\mathrm{t}_{\mathrm{k}}\right)$. Para a mudança de referencial das coordenadas, quando couber, o primeiro passo é realizar a atualização dos parâmetros de transformação da época $\left(\mathrm{t}_{\mathrm{k}}\right)$ para a época $\left(\mathrm{t}_{0}\right)$ das coordenadas no sistema de referência origem. E, em seguida, de posse das velocidades da estação, realiza-se a atualização das coordenadas da época $\left(\mathrm{t}_{0}\right)$ para a época $(\mathrm{t})$.

\subsection{Atualização de Parâmetros, Transformação de Referencial e Atualização de Coordenadas}

A atualização de parâmetros de transformação de uma época $t_{k}\left(P\left(t_{k}\right)\right)$ para uma época $t_{0}\left(P\left(t_{0}\right)\right)$ das coordenadas no sistema origem, pode ser realizada a partir da Expressão 1 (ITRF, 2015a; Monico, 2008):

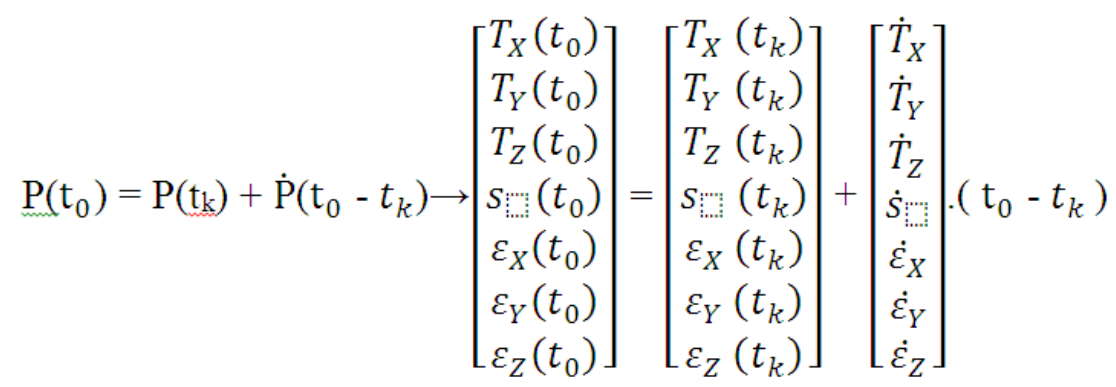

Onde P é o vetor das variações temporais dos parâmetros. Após a atualização destes, realiza-se a transformação entre os sistemas de referência, através da transformação de Helmert, por meio de três translações, três rotações e um fator de escala. O modelo matemático para transformar as coordenadas no sistema origem (A) para o sistema destino (B) é dado pela Expressão 2:

$$
\left[\begin{array}{l}
\mathrm{X} \\
\mathrm{Y} \\
\mathrm{Z}
\end{array}\right]_{\mathrm{B}\left(\mathrm{t}_{0}\right)}=\left[\begin{array}{l}
\mathrm{X} \\
\mathrm{Y} \\
\mathrm{Z}
\end{array}\right]_{\mathrm{A}\left(\mathrm{t}_{0}\right)}+\left[\begin{array}{l}
\mathrm{T}_{\mathrm{X}} \\
\mathrm{T}_{\mathrm{Y}} \\
\mathrm{T}_{\mathrm{Z}}
\end{array}\right]_{\mathrm{t}_{0}}+\left[\begin{array}{llr}
\mathrm{S} & -\varepsilon_{\mathrm{z}} & \varepsilon_{\mathrm{y}} \\
\varepsilon_{\mathrm{z}} & \mathrm{s} & -\varepsilon_{\mathrm{X}} \\
-\varepsilon_{\mathrm{y}} & \varepsilon_{\mathrm{X}} & \mathrm{S}
\end{array}\right]_{\mathrm{t}_{0}} \cdot\left[\begin{array}{l}
\mathrm{X} \\
\mathrm{Y} \\
\mathrm{Z}
\end{array}\right]_{\mathrm{A}\left(\mathrm{t}_{0}\right)}
$$

Todos os elementos da Expressão 2 já foram descritos. É importante salientar que os sete parâmetros de transformação e as coordenadas dos pontos nos dois sistemas devem estar na mesma época.

Por fim, com as coordenadas no sistema de referência de interesse e com os vetores de velocidades, pode-se aplicar a Expressão 3 para se obter as coordenadas atualizadas no referencial desejado.

$$
\left[\begin{array}{l}
\mathrm{X} \\
\mathrm{Y} \\
\mathrm{Z}
\end{array}\right]_{\mathrm{B}(\mathrm{t})}=\left[\begin{array}{l}
\mathrm{X} \\
\mathrm{Y} \\
\mathrm{Z}
\end{array}\right]_{\mathrm{B}\left(\mathrm{t}_{0}\right)}+\left[\begin{array}{c}
\mathrm{V}_{\mathrm{X}} \\
\mathrm{V}_{\mathrm{Y}} \\
\mathrm{V}_{\mathrm{Z}}
\end{array}\right]_{\mathrm{B}}\left(\mathrm{t}-\mathrm{t}_{0}\right)
$$

Em que t é a época de interesse e to é a época das coordenadas a serem atualizadas. 
Para se obter o vetor velocidade da estação de interesse, na região de abrangência do SIRGAS2000, recomenda-se a utilização do modelo de velocidade VEMOS2009. Porém, salienta-se que há disponível pelo menos 14 modelos de velocidade.

\subsection{Modelos de Velocidade Para a Placa Sul-Americana}

Segundo Sapucci e Monico (2000), a determinação do movimento das placas litosféricas que compõem a parte superior da Terra justifica o rigor com que deve ser tratada uma transformação entre diferentes referenciais de alta precisão. Os movimentos das placas geram alterações nas coordenadas das estações com o passar do tempo, de tal forma que uma simples transformação de Helmert não é suficiente, sendo necessário considerar as variações dos parâmetros envolvidos nessa transformação.

Salienta-se que existem vários modelos de campo de velocidade disponíveis, inclusive modelos globais. Alguns desses modelos utilizam a condição NNR (No-Net-Rotation). Esta condição considera que o momento angular total de todas as placas tectônicas deve ser zero (Solomon e Sleep, 1974). Com isso, não há impacto nas velocidades relativas da placa, sendo uma condição adicional utilizada para definir uma referência para os movimentos de placa que não estão ligados a uma placa particular.

Com relação aos modelos de campo de velocidade disponibilizados para placa litosférica SulAmericana, destaca-se o modelo VEMOS2009, que é o modelo recomendado pelo SIRGAS. Salienta-se que o VEMOS é referenciado ao ITRF2005(época 2000,0). Este modelo de velocidade está vinculado ao ITRF2005 e corresponde a uma grade espaçadas de $1^{\circ}$ com velocidades horizontais, as quais podem ser interpoladas pelo programa VMS2009 disponível no sítio (http://www.sirgas.org/index.php?id=54) do SIRGAS (Drewes e Heidbach, 2012).

A precisão média alcançada na estimação das velocidades com esse modelo é cerca de 1,5 mm/ano (Sánchez et al., 2013). Porém, de acordo com Drewes e Heidbach (2012), os erros das velocidades variam dependendo da região, podendo variar de $\pm 1 \mathrm{~mm} / \mathrm{ano}$ até $\pm 9 \mathrm{~mm} / \mathrm{ano}$ em áreas de cobertura com observações esparsas.

A UNAVCO (University NAVstar COnsortiu) disponibiliza 14 modelos de campo de velocidade para calcular o movimento da placa litosférica Sul-Americana. Estes modelos permitem calcular online a velocidade das estações de interesse (UNAVCO, 2014). São disponíveis os seguintes modelos:

- APKIM2000: Foram calculadas as velocidades de 12 placas tectônicas tendo como base a análise dos dados de VLBI, SLR e GPS;

- APKIM2005-DGFI: Foram calculadas as velocidades de 17 placas tectônicas com base em observações espaciais geodésicos (GPS, SLR, DORIS, VLBI), calculado pela DGFI;

- APKIM2005-IGN (APKIM2005 - Institute Géographique National ): Foram calculadas as velocidades de 17 placas tectônicas. O modelo utiliza como entradas de dados as velocidades das estações horizontais de solução ITRF2005 do IGN;

- CGPS 2004 (Continuous GPS 2004): Foram estimadas as velocidades de 18 placas tectônicas tendo como base a análise de 106 estações GPS de operações contínua distribuída globalmente, incluído 12 anos de dados de 1991 a 2003;

- GEODVEL 2010 (GEODesy VELocity 2010): Foram estimadas as velocidades de 11 placas tectônicas, usando 4 técnicas de observações geodésicas (GPS, VLBI SLR e DORIS). Foi determinada também a velocidade do centro da Terra; 
- GSRM v1.2 2004 (Global Strain Rate Map 2004): Foram calculadas as velocidades de 25 placas tectônicas;

- HS2-NUVEL 1A (HotSpot2 Northwestern University VELocity model 1A ): Foram calculadas as velocidades de 14 placas tectônicas e calculadas as taxas de variações e o azimute do deslocamento de cada placa;

- HS3-NUVEL 1A (HotSpot3 Northwestern University VELocity model 1A): Foram calculadas as velocidades de 15 placas tectônicas. São calculados as taxas de variações e o azimute do deslocamento de cada placa;

- ITRF2000-AS\&B: Foram estimadas as velocidades de 6 placas tectônicas, utilizando SLR, LLR, DORIS, VLBI e GPS;

- ITRF2000-D\&A: Foram estimadas as velocidades de 13 placas tectônicas. Com a utilização de aproximadamente 350 velocidades de estações;

- MORVEL 2010 (Mid Ocean Ridge VELocity 2010): Foram estimadas as velocidades de 28 placas tectônicas que ocupa $97 \%$ da superfície da Terra. Este modelo foi determinado a partir de dados geológicos e de dados GPS;

- NUVEL 1: Foram estimadas as velocidades de 14 placas tectônicas. Foram obtidos a partir de dados geológicos e geofísicos;

- NUVEL 1A: Foram estimadas as velocidades de 15 placas tectônicas. As velocidades foram obtidas a partir de dados geológicos e geofísicos;

- REVEL-2000: Foram estimadas as velocidades de 18 placas tectônicas. O modelo é derivado de dados de geodésia espacial (principalmente GPS) no período de 1993 a 2000.

Com exceção dos modelos de velocidade GEODVEL 2010, HS2-NUVEL 1A e HS3-NUVEL 1A, todos utilizam o conceito de NNR.

\section{Metodologia}

Neste trabalho foram obtidos dados de 86 estações disponíveis pela RBMC no dia 15/07/2013 (DOY 196). Os dados GNSS (período de coleta de 24 horas) foram processados no IBGE-PPP, e as coordenadas estimadas estão referenciadas ao IGb08 (ITRF2008), época 2013,54. Em seguida, as coordenadas de referência da RBMC, obtidas no descritivo das estações, foram transformadas e atualizadas do SIRGAS2000 (ITRF2000), época 2000,4 para o IGb08 (ITRF2008), época 2013,54. Desta forma, as coordenadas comparadas estão no mesmo sistema e época de referência. Salienta-se que foram utilizados 3 conjuntos de parâmetros de transformação, descritos no tópico 2, em conjunto com 15 modelos de velocidade, descritos no tópico 3.2, totalizando, desta forma, 45 possibilidades de transformação de referencial e atualização de coordenadas.

Todas as análises foram realizadas em coordenadas geodésicas curvilíneas ( $\phi$ e $\lambda)$. Na sequência foram calculadas as discrepâncias entre as coordenadas estimadas, em IGb08, época 2013,54, e as coordenadas de referência, transformadas e atualizadas para o IGb08, época 2013,54.

É importante destacar que as discrepâncias altimétricas não serão apresentadas, pois o processo de atualização de coordenadas é predominantemente horizontal. Em outras palavras, o processo de atualização de coordenadas não tem influência significativa na componente altimétrica. As discrepâncias em latitudes e longitudes foram calculadas da seguinte forma (em radianos) (Expressões 4 e 5): 


$$
\begin{gathered}
\Delta \phi_{\mathrm{rad}}=\phi_{\text {Est }}-\phi_{\mathrm{Ref}} \\
\Delta \lambda_{\mathrm{rad}}=\lambda_{\text {Est }}-\lambda_{\mathrm{Ref}}
\end{gathered}
$$

Sendo $\phi_{\text {Est }}$ e $\lambda_{\text {Est }}$ as coordenadas estimadas em IGb08, época 2013,54, e $\phi$ Ref e $\lambda$ Ref as coordenadas de referência transformadas e atualizadas para o IGb08, época 2013,54.

Para conversão das discrepâncias em metros, foram utilizadas as seguintes Expressões 6 e 7:

$$
\begin{gathered}
\Delta \phi_{(\mathrm{m})}=\Delta \phi_{(\mathrm{rad})} * \mathrm{M} \\
\Delta \lambda_{(\mathrm{m})}=\Delta \lambda_{(\mathrm{rad})} * \mathrm{~N} * \cos (\phi)
\end{gathered}
$$

sendo,

$M=$ raio de curvatura da seção meridiana;

$\mathrm{N}=$ raio de curvatura da seção primeira vertical;

$\phi=$ latitude média do ponto.

$\mathrm{Na}$ sequência forma calculados as discrepâncias planimétricas $\left(\mathrm{D}_{\mathrm{p}}(\mathrm{m})\right.$ ) da seguinte forma Expressão 8:

$$
\mathrm{D}_{\mathrm{p}(\mathrm{m})}=\sqrt{\Delta \phi_{(\mathrm{m})}^{2}+\Delta \lambda_{(\mathrm{m})}^{2}}
$$

Com as precisões fornecidas em metros no IBGE-PPP, foram calculadas as precisões planimétricas $\left(\sigma_{\mathrm{P}(\mathrm{m})}\right)$ da seguinte forma Expressão 9:

$$
\sigma_{\mathrm{p}(\mathrm{m})}=\sqrt{\sigma_{\phi(\mathrm{m})}^{2}+\sigma_{\lambda(\mathrm{m})}^{2}}
$$

Sendo $\sigma_{\phi(\mathrm{m})}$ a precisão na latitude em metros e $\sigma_{\lambda(m)}$ a precisão em longitude, também em metros. Por fim, após os cálculos das discrepâncias e precisões planimétricas, foi possível calcular a acurácia planimétrica $\left(\mathrm{A}_{\mathrm{p}}(\mathrm{m})\right)$, proposta por Gauss e dada por Mikhail e Ackerman (1976), Expressão 10:

$$
\mathrm{A}_{\mathrm{p}(\mathrm{m})}=\sqrt{\mathrm{D}_{\mathrm{p}(\mathrm{m})}^{2}+\sigma_{\mathrm{p}(m)}^{2}}
$$

É importante destacar que os dados GNSS processados no IBGE-PPP correspondem a um período de 24 horas, com isso, a precisão esperada no processamento é milimétrica. Desta forma, as acurácias planimétricas calculadas, foram mais influenciadas pelas discrepâncias do que pela precisão. Além disso, destaca-se também que no momento em que os dados foram processados (15/07/2013) o IBGE-PPP não processava dados GLONASS. Desta forma, somente foram processados dados GPS no IBGE-PPP. Porém, salienta-se que isto faria pouca diferença nos resultados, visto que a integração entre o GPS e o GLONASS no PPP é significativa para intervalos curtos de rastreio de dados GNSS.

O fluxograma ilustrado na Figura 1 sumariza o procedimento metodológico descrito anteriormente e adotado neste trabalho. 


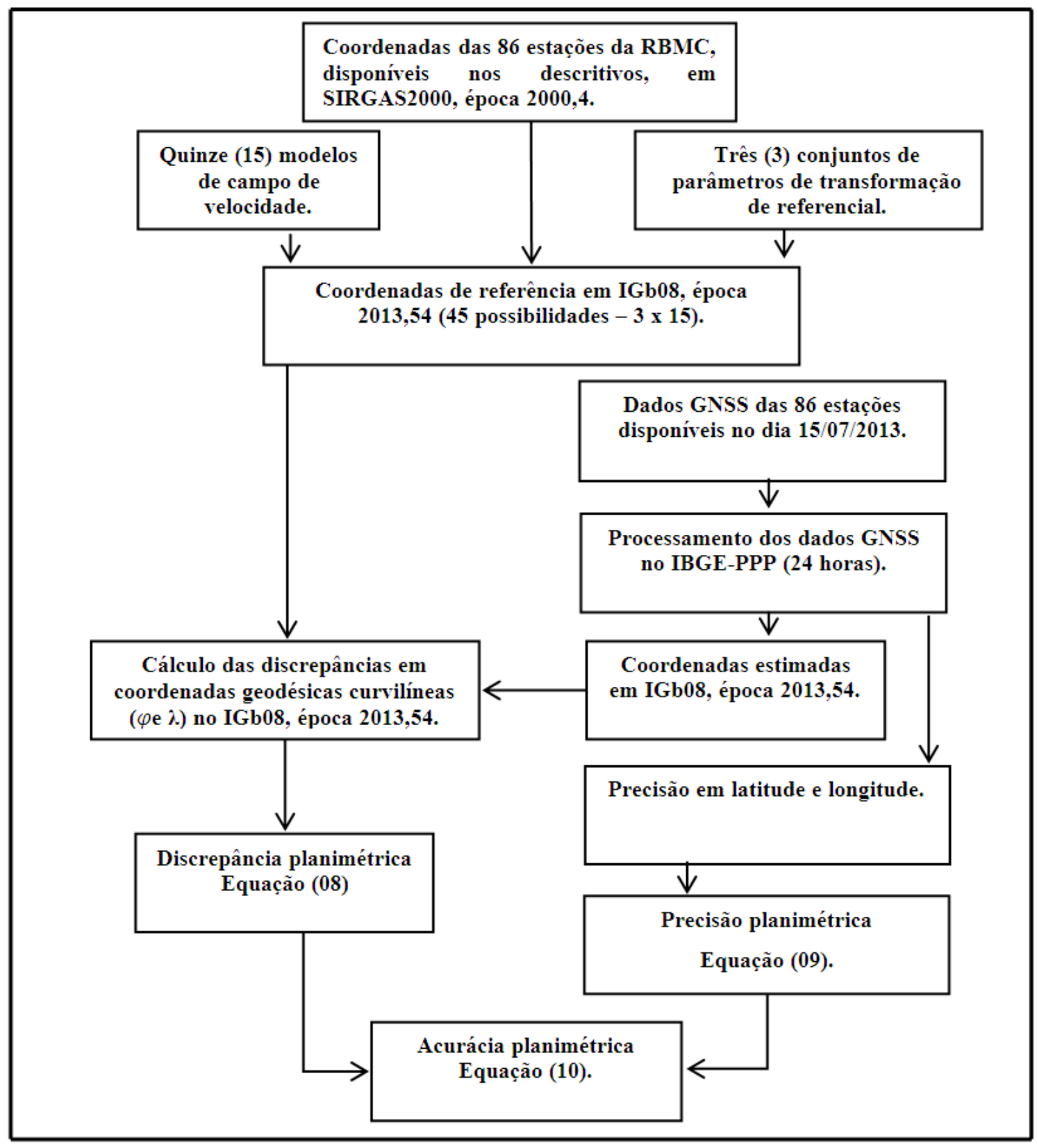

Figura 1: Fluxograma da metodologia realizada no trabalho.

As figuras 2, 3 e 4 ilustram as três estratégias de transformação utilizadas neste trabalho, sendo que para cada uma delas, foram utilizados 15 modelos de velocidades, totalizando as 45 possibilidades. Destaca-se que, em nenhuma das três s apresentadas foi considerada a mudança no referencial de velocidade. Porém, foram realizados testes com o modelo de velocidade VEMOS, que estima as velocidades referenciadas ao ITRF2005. As diferenças encontradas foram da ordem de poucos milímetros, podendo ser, na prática, negligenciadas. As mesmas considerações foram realizadas por Carvalho et al. (2015). 


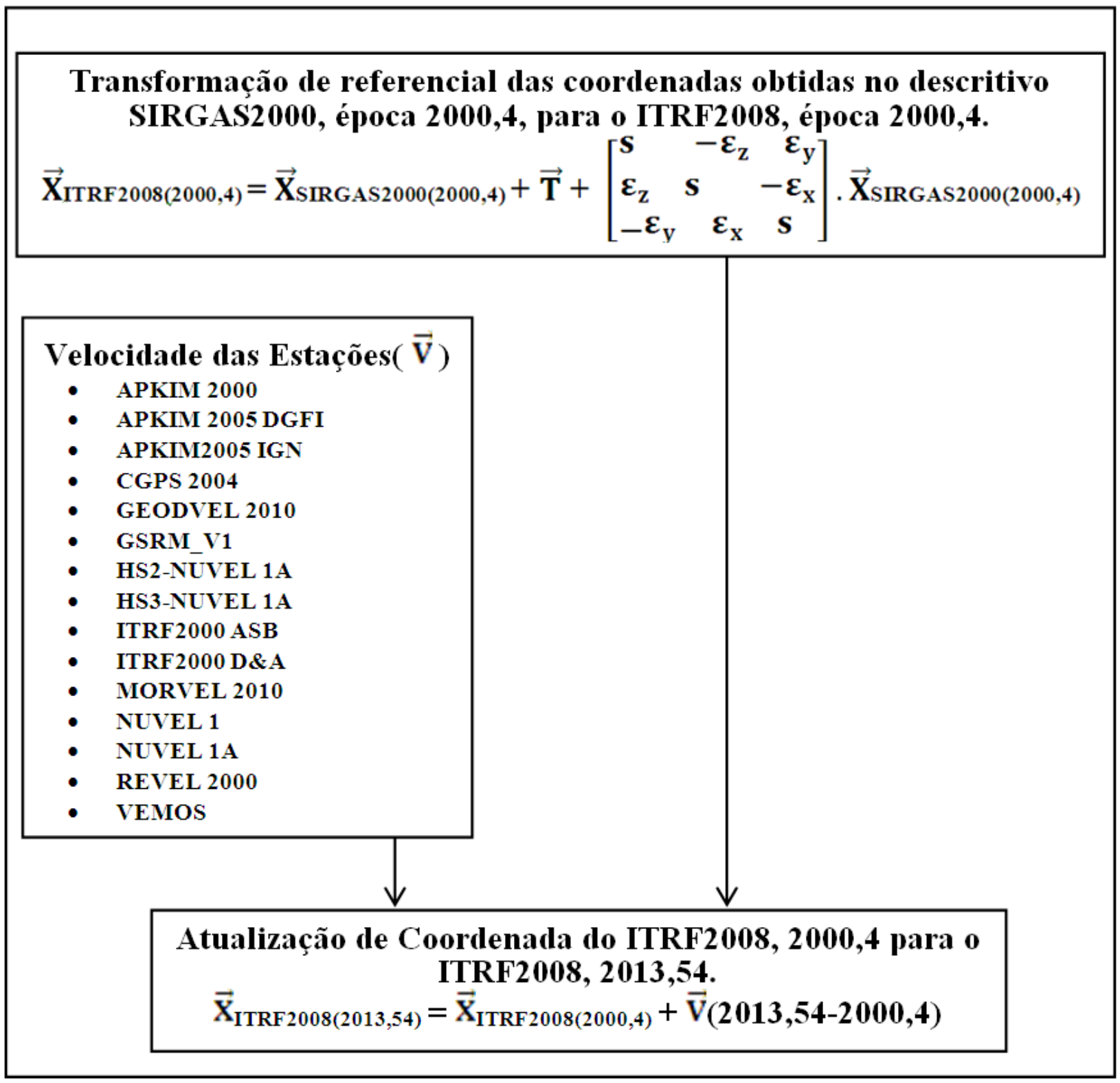

Figura 2: Fluxograma da estratégia de transformação utilizando os parâmetros do IBGE. 


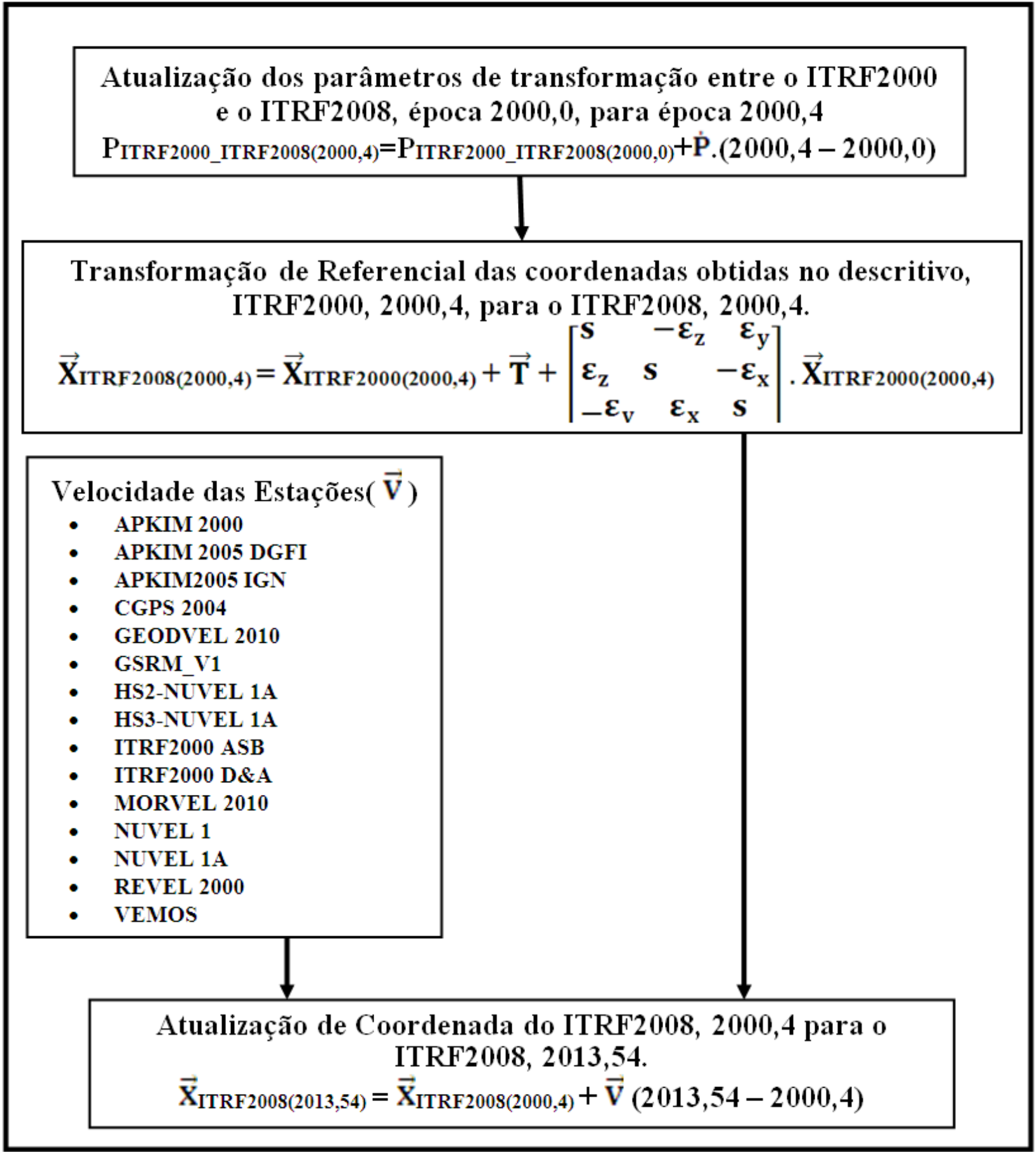

Figura 3: Fluxograma da estratégia de transformação utilizando os parâmetros disponibilizados por Petit e Luzum (2010). 
Atualização dos parâmetros de transformação entre os ITRF2000

e o ITRF2005, época 2000,0 , para época 2000,4

$P_{\text {ITRF2000_ITRF2005(2000,4) }}=$ PITRF2000_ITRF2005(2000,0) + P..$(2000,4-2000)_{1}$

Transformação de Referencial das coordenadas obtidas no descritivo ITRF2000, 2000,4, para o ITRF2005, $2000,4$.

$$
\overrightarrow{\mathrm{X}}_{\mathrm{ITRF} 2005(2000,4)}=\overrightarrow{\mathrm{X}}_{\mathrm{ITRF} 2000(2000,4)}+\overrightarrow{\mathrm{T}}+\left[\begin{array}{llr}
S & -\varepsilon_{\mathrm{z}} & \varepsilon_{\mathrm{y}} \\
\varepsilon_{\mathrm{z}} & \mathrm{s} & -\varepsilon_{\mathrm{X}} \\
-\varepsilon_{\mathrm{V}} & \varepsilon_{\mathrm{X}} & \mathrm{S}
\end{array}\right] . \overrightarrow{\mathrm{X}}_{\mathrm{ITRF2000(2000,4)}}
$$

Atualização dos parâmetros de transformação entre os ITRF2005 e

o ITRF2008, época 2005,0, para época 2000,4.

PITRF2005_ITRF2008(2000,4) $_{\text {PITRF2005_ITRF2008(2005,0) }+ \text { P. }(2000,4-2005,0)}$

Transformação de Referencial das coordenadas do ITRF2005, época 2000,4 , para o ITRF2000, época 2000,4 .

$\overrightarrow{\mathrm{X}}_{\mathrm{ITRF} 2008(2000,4)}=\overrightarrow{\mathrm{X}}_{\mathrm{ITRF} 2005(2000,4)}+\overrightarrow{\mathrm{T}}+\left[\begin{array}{llr}\mathrm{S} & -\varepsilon_{\mathrm{z}} & \varepsilon_{\mathrm{y}} \\ \varepsilon_{\mathrm{z}} & \mathrm{S} & -\varepsilon_{\mathrm{X}} \\ -\varepsilon_{\mathrm{y}} & \varepsilon_{\mathrm{x}} & \mathrm{S}\end{array}\right] . \overrightarrow{\mathrm{X}}_{\mathrm{ITRF} 2005(2000,4)}$

Velocidade das Estações( $\vec{V})$

- APKIM 2000

- APKIM 2005 DGFI

- APKIM2005IGN

- CGPS 2004

- GEODVEL 2010

- GSRM V1

- HS2-NúVEL 1A

- HS3-NUVEL 1A

- ITRF2000 ASB

- ITRF2000 DA

- MORVEL 2010

- NUVEL 1

- NUVEl $1 \mathrm{~A}$

- REVEL 2000

- VEMOS

Figura 4: Fluxograma da estratégia de transformação utilizando os parâmetros disponibilizados por Altamimi et al.(2007) e Petit e Luzum (2010).

\section{Resultados e Discussão}

A Tabela 3 mostra as médias das acurácias na componente planimétrica $(\varphi, \lambda)$ para cada uma das possibilidades de transformação de referencial e atualização de coordenadas. Foram inseridas entre parênteses as colocações das possibilidades. Por exemplo, a combinação entre os parâmetros do IBGE e o modelo de velocidades APKIM 2000 ficou classificado como a pior combinação (quadragésima quinta). 
Tabela 3: Médias das acurácias planimétricas $(\varphi, \lambda)$, para as 45 possibilidades de transformação de referencial e atualização de coordenadas.

\begin{tabular}{|c|c|c|c|}
\hline \multicolumn{4}{|c|}{$\begin{array}{c}\text { POSSIBILDADES DE TRANSFORMAÇ̃̃O DE } \\
\text { REFERENCIAL E AT UALIZAĊ̃̃O DE COORDE NADAS }\end{array}$} \\
\hline \multirow{2}{*}{$\begin{array}{l}\text { MODE LOS DE } \\
\text { VELOCDADE }\end{array}$} & \multicolumn{3}{|c|}{ CONJUNTODE PARÄMIE TROSDE TRANSFORMACÃO } \\
\hline & IBGE & Petit e Luzum (2010) & $\begin{array}{l}\text { Altamimi et al. (2007) e } \\
\text { Petit e Luzum (2010) }\end{array}$ \\
\hline APKIM 2000 & $0,049 \mathrm{~m}(45)$ & $0,016 \mathrm{~m}(05)$ & $0,015 \mathrm{~m}(04)$ \\
\hline APKIM 2005 DGFI & $0,025 \mathrm{~m} \mathrm{(27)}$ & $0,022 \mathrm{~m}(15)$ & $0,023 \mathrm{~m} \mathrm{(22)}$ \\
\hline APKIM2005 IGN & $0,015 \mathrm{~m}(03)$ & $0,035 \mathrm{~m}(41)$ & $0,036 \mathrm{~m}(42)$ \\
\hline CGPS 2004 & $0,031 \mathrm{~m} \mathrm{(36)}$ & $0,016 \mathrm{~m}(06)$ & $0,017 \mathrm{~m} \mathrm{(08)}$ \\
\hline GEODVEL 2010 & $0,021 \mathrm{~m}(14)$ & $0,022 \mathrm{~m}(16)$ & $0,023 \mathrm{~m} \mathrm{(23)}$ \\
\hline GSRM V1 & $0,023 \mathrm{~m}(19)$ & $0,025 \mathrm{~m}(28)$ & $0,026 \mathrm{~m}(32)$ \\
\hline HS2-NÜVEL $1 \mathrm{~A}$ & $0,032 \mathrm{~m}(37)$ & $0,025 \mathrm{~m}(29)$ & $0,025 \mathrm{~m}(30)$ \\
\hline HS3-NUVEL 1A & $0,034 \mathrm{~m}(39)$ & $0,023 \mathrm{~m} \mathrm{(20)}$ & $0,023 \mathrm{~m} \mathrm{(24)}$ \\
\hline ITRF2000 ASB & $0,028 \mathrm{~m} \mathrm{(35)}$ & $0,022 \mathrm{~m}(17)$ & $0,022 \mathrm{~m}(18)$ \\
\hline ITRF2000 DA & $0,020 \mathrm{~m}(11)$ & $0,024 \mathrm{~m}(26)$ & $0,025 \mathrm{~m}(31)$ \\
\hline MORVEL 2010 & $0,042 \mathrm{~m} \mathrm{(43)}$ & $0,014 \mathrm{~m}(01)$ & $0,014 \mathrm{~m} \mathrm{(02)}$ \\
\hline NUVEL 1 & $0,033 \mathrm{~m} \mathrm{(38)}$ & $0,027 \mathrm{~m}(33)$ & $0,027 \mathrm{~m}(34)$ \\
\hline NUVEL $1 \mathrm{~A}$ & $0,034 \mathrm{~m}(40)$ & $0,023 \mathrm{~m} \mathrm{(21)}$ & $0,023 \mathrm{~m} \mathrm{(24)}$ \\
\hline REVEL 2000 & $0,042 \mathrm{~m}(44)$ & $0,020 \mathrm{~m}(12)$ & $0,020 \mathrm{~m}(13)$ \\
\hline VEMOS & $0,019 \mathrm{~m}(10)$ & $0,017 \mathrm{~m}(07)$ & $0,018 \mathrm{~m} \mathrm{(09)}$ \\
\hline
\end{tabular}

De acordo com a Tabela 3, verifica-se que, com a utilização dos parâmetros do IBGE, na média, a melhor opção é usar o modelo de velocidade APKIM 2005 IGN (possibilidade 03), seguido pelo modelo de velocidade VEMOS. Porém, a diferença entre ambos é de apenas $4 \mathrm{~mm}$. Contudo, ao analisar todos os modelos de velocidade, comparando o melhor caso (APKIM 2005 IGN) com o pior caso (APKIM 2000 - possibilidade 45), a diferença aumenta para $3,4 \mathrm{~cm}$. Ou seja, a escolha de modelo de velocidade no processo de atualização de coordenadas pode ter impacto centimétrico no PPP.

Ao analisar o segundo e o terceiro grupo de parâmetros de transformação de referencial, verificase na média, diferenças de apenas $1 \mathrm{~mm}$ entre ambos. Destaca-se que essas diferenças ocorrem em 7 casos, sendo que nos 8 casos restantes não ocorrem diferenças na ordem de milímetros. Portanto, pode-se dizer que não há diferenças entre os dois grupos na prática. Contudo, ao comparar as diferenças com o grupo de parâmetros do IBGE, verificam-se alterações significativas, como por exemplo, no melhor caso (com relação ao parâmetro do IBGE), a acurácia média foi de apenas $1,5 \mathrm{~cm}$, enquanto que nos outros casos aumentou para $3,5 \mathrm{~cm}$.

Destaca-se também que o pior caso (APKIM2000 - possibilidade 45), com relação ao parâmetro do IBGE, atingiu 4,9 cm, sendo um dos melhores casos quando comparado à utilização dos outros conjuntos de parâmetros. Com exceção do grupo de parâmetros do IBGE, o modelo MORVEL2010 se destacou, apresentando acurácia média igual a apenas 1,4 cm, proporcionando os melhores resultados.

Todos os valores de acurácia analisados neste trabalho (Tabela 3) se referem a um valor médio calculado para todas as estações da RBMC, que são distribuídas ao longo do território brasileiro. Desta forma, não foi possível verificar a acurácia de cada possibilidade em regiões específicas do Brasil.

Desta forma, verificou-se a eficiência de cada possibilidade de transformação de referencial e atualização de coordenadas por região, como mostram as Tabelas 4 e 5. A Tabela 4 mostra as possibilidades que foram responsáveis pelos menores valores de acurácias planimétrica, por região do Brasil, e a Tabela 5 mostra os maiores valores. As possibilidades indicadas nas tabelas indicam a classificação média para o Brasil, para fins de comparação. 
Tabela 4: Melhores possibilidades de transformação de referencial e atualização de coordenadas para cada região do Brasil.

\begin{tabular}{|c|c|c|c|c|c|}
\hline & \multirow{2}{*}{\multicolumn{5}{|c|}{ RE GIÕES }} \\
\hline & & & & & \\
\hline & NORDE STE & NORTE & CENT RO OE STE & SUDE STE & SUL \\
\hline No DE E ST AC̣ÕES & 22 & 19 & 10 & 27 & 8 \\
\hline POSSIBILIDADE & 3 & 6 & 6 & 1 & 11 \\
\hline PARÂMETROS & IBGE & Petit e L uzum (2010) & Petit e Luzum (2010) & Petit eL uzum (2010) & IBGE \\
\hline MODELO & $\begin{array}{l}\text { APKIML2005 } \\
\text { IGN }\end{array}$ & CGPS 2004 & CGPS 2004 & MORVEL & IT RF 2000 D A \\
\hline MÉDIA & $0,009 \mathrm{~m}$ & $0,016 \mathrm{~m}$ & $0,012 \mathrm{~m}$ & $0,011 \mathrm{~m}$ & $0,011 \mathrm{~m}$ \\
\hline
\end{tabular}

Tabela 5: Piores possibilidades de transformação de referencial e atualização de coordenadas para cada região do Brasil.

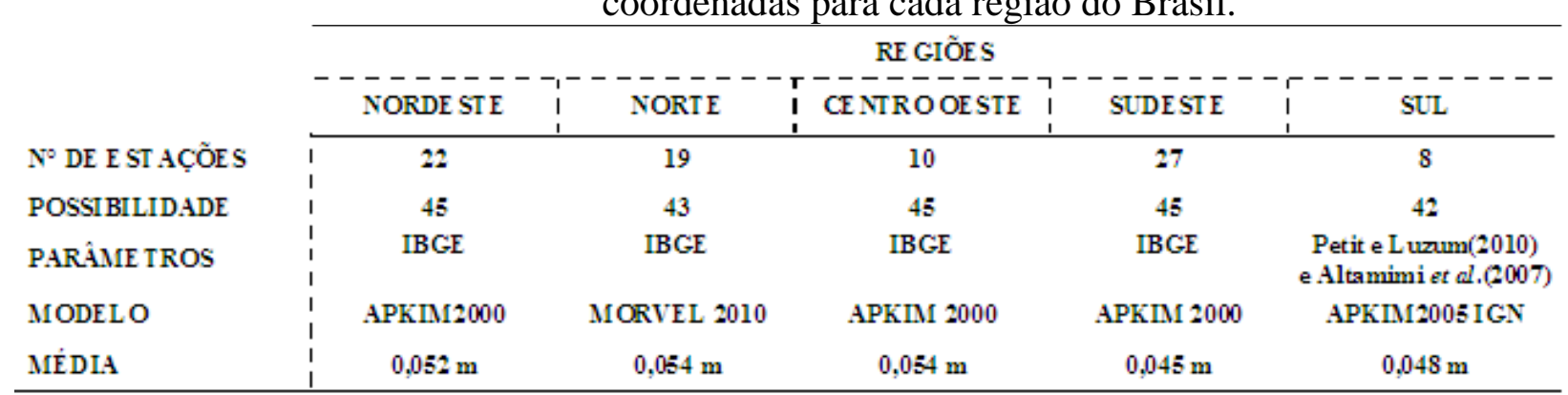

É interessante notar, de acordo com a Tabela 4, que a possibilidade 01 (que na média é melhor para o Brasil), na média, é a melhor opção apenas para o sudeste. Porém, esta região é a que apresenta o maior número de estações (27 no total), distribuídas em uma área consideravelmente menor do que a região nordeste, que tem a segunda maior concentração de estações (22 estações). A décima primeira melhor possibilidade (na média para o Brasil) foi a melhor opção para a região Sul do país, que conta com apenas 8 estações. Destaca-se que o modelo VEMOS, utilizado pelo IBGE-PPP não compareceu em nenhuma combinação referente as melhores possibilidades para cada região do Brasil. Em contrapartida, os parâmetros utilizados pelo IBGEPPP foram responsáveis pelos melhores resultados em duas regiões do Brasil (regiões Sul e Nordeste). No caso da região nordeste, a combinação entre os parâmetros utilizados pelo IBGEPPP e o modelo de velocidade APKIN 2005 IGN proporcionou acurácia média de ordem milimétrica $(9 \mathrm{~mm})$.

Ao analisar a Tabela 5, percebe-se que o modelo de velocidade APKIM2000 juntamente com o conjunto de parâmetros do IBGE, não é uma boa opção na atualização de coordenadas e transformação de referencial nas regiões Nordeste, Centro-Oeste e Sudeste.

\section{Considerações Finais e Conclusões}

De acordo com os resultados obtidos, verifica-se o bom desempenho do modelo de velocidades VEMOS, na média, para o Brasil, pois independentemente do parâmetro de transformação de referencial utilizado, apresentou resultados satisfatórios (sétima, nona e décima melhores possibilidades). Contudo, nas seis melhores possibilidades em nenhuma combinação o VEMOS foi utilizado. Deve-se salientar que o modelo de velocidades MORVEL apresentou desempenho melhor do que o VEMOS em conjunto com os parâmetros de transformação (com exceção dos 
parâmetros do IBGE), proporcionando as duas melhores combinações (Petit e Luzum (2010) MORVEL 2010; Altamimi et al. 2007 e Petit e Luzum (2010) - MORVEL 2010). Porém, ao utilizar o modelo de velocidades MORVEL 2010 em conjunto com os parâmetros disponibilizados pelo IBGE, a acurácia do processo de transformação de referencial e atualização de coordenadas foi degradada de forma significativa (quadragésima terceira melhor combinação).

Destaca-se que ao analisar as possibilidades de transformação de referencial e atualização de coordenadas para cada região do Brasil, a melhor combinação na média, para o Brasil (Petit e Luzum (2010) - MORVEL 2010), foi a melhor possibilidade somente para a região Sudeste, que possui mais de $30 \%$ das estações analisadas. Além disso, a décima primeira melhor combinação (IBGE-ITRF2000 DA) na média para o Brasil, foi a melhor opção para a região Sul do Brasil. Porém, esta região possui apenas 8 estações da RBMC (menor quantidade de estações), ao contrário da região sudeste, que apresenta a maior quantidade de estações da RBMC (27 estações). Verifica-se, diante do exposto, que para cada região do Brasil há uma combinação diferente que proporciona os melhores resultados.

Por fim, destaca-se que a estratégia de transformação na qual se emprega os parâmetros utilizados no IBGE-PPP em conjunto com o modelo de velocidade VEMOS2009 apresentou, na média, a décima melhor combinação para o Brasil. Porém, deve-se ter cuidado na escolha do modelo de velocidade a ser empregado em concomitância com os parâmetros do IBGE-PPP, pois das 11 piores combinações, 9 foram proporcionadas com o uso dos parâmetros do IBGE.

\section{REFERÊNCIAS BIBLIOGRÁFICAS}

Altamimi, Z.; Xavier, C.; Laurent, M. ITRF2008: an improved solution of the international terrestrial reference frame. Journal of Geodesy (2011). 85:457-473. DOI 10.1007/s00190-0110444-4.

Altamimi, Z.; Collilieux, X.; Legrand, J.; Garayt, B.; Boucher C. (2007), ITRF2005: A new release of the International Terrestrial Reference Frame based on time series of station positions and Earth Orientation Parameters. J. Geophys. Res.,112, B09401, DOI:10.1029/2007JB004949.

Carvalho, A. S.; Dal Poz. W. R.; Gripp, J.; Larocca. A. P. C.; Krueger. C. P. Sistematização das etapas para determinação posicional em SIRGAS2000 com base nas coordenadas ITRF(IGB08) obtidas por serviços PPP online. Revista Brasileira de Cartografia (2015) N 67/1 43-58. ISSN: 1808-0936.

Drewes, H.; Heidbach, O. (2012). The 2009 Horizontal Velocity Field for South America and the Caribbean. In: Kenyon S., M.C. Pacino, U. Marti (Eds.), "Geodesy for Planet Earth", IAG Symposia, 136: 657-664.

EPNCB (2014). Guidelines for EUREF Densifications. Disponível em: < ftp://epncb. oma.be/pub/general/Guidelines_for_EUREF_Densifications.pdf >. Acesso em: 18 jun 2014.

IBGE (2013). Manual do Usuário Aplicativo Online IBGE-PPP Versão: dezembro 2013. Disponível em:< http://www.ibge.gov.br/home/geociencias/geodesia/ppp/manual_ppp.pdf > , acesso em 14 novembro de 2014.

IBGE (2015a). Posicionamento por Ponto Preciso (PPP). Disponível em: < http://www.ppp.ibge.gov.br/ppp.htm>. Acesso em: 07 jul 2015. 
IBGE (2015b). Término do período de transição para adoção no Brasil do Sistema de Referência Geocêntrico para as Sméricas (SIRGAS), em sua realização de 2000,4 (SIRGAS2000). Disponível em: <ftp://geoftp.ibge.gov.br/documentos/geodesia/pdf/nota_tecnica_termino_periodo_transicao_sirg as2000.pdf>. Acesso em: 16 jun 2015.

ITRF (2015a). Transformation parameters. Disponível em: <http://itrf.ensg.ign.fr/trans_para.php> Acesso em: 16 jan. 2015.

Mikhail, E.; Ackerman, F.; Observations and Least Squares. University Press of America, 1976.

Monico, J. F. G. Posicionamento pelo GNSS: descrição, fundamentos e aplicações. São Paulo: Ed. da Unesp, 2008.

Petit, G.; Luzum, B. IERS Conventions (2010) - IERS Technical Note 36. IERS. Frankfurt am Main: Verlag des Bundesamts für Kartographie und Geodäsie, p. 179. 2010.

Sánchez, L.; Seemüller, W.; Drewes, H.; Mateo, L.; González, G.. Silva, A.; Pampillón, J.; Martinez, W.; Cioce, V.; Cisneros, D.; Cimbaro, S. Long-term stability of the SIRGAS reference frame and episodic station movements caused by the seismic activity in the SIRGAS region. In: Altamimi Z.; Collilieux, X. (Eds.) Reference Frames for Applications in Geosciences, IAG Symposia 138: 153-161, Springer, 10.1007/978-3-642-32998-2_24, 2013.

Sapucci, L. F., Monico, J. F. G. Transformação de Helmert generalizada no posicionamento de alta precisão: fundamentação teórica e exemplificações. Ver. Bras. Geof. Vol. 18 no 2. São Paulo, 2000.

SIRGAS (2015). SIRGAS 2000. Disponível em: < http://www.sirgas.org/index.php?id=77> Acesso em: fev 2015.

Solomon, S. C.; Sleep, N. H. Some physical models for absolute plate motions, J. Geophys (1974). Res., 79, $2557-2567$

UNAVCO (2014). Plate Motion Calculator. Disponível em: < http://www.unavco.org/software/geodetic-utilities/plate-motion-calculator/plate-motioncalculator.html >. Acesso em: 19 set 2014.

Recebido em Julho de 2015.

Aceito em Janeiro de 2016. 